\title{
Mycobacterial 19-kDa lipoprotein mediates Mycobacterium tuberculosis-induced apoptosis in monocytes/ macrophages at early stages of infection
}

Dear Editor,

An increasing number of bacterial pathogens have been identified as mediators of apoptosis ${ }^{1}$ and apoptosis has also been described in monocytes/macrophages following in vivo and in vitro Mycobacterium tuberculosis (MTB) infection. ${ }^{2,3}$ This process is directly related to multiplicity of infection (MOI), occurring at the very early stages of in vitro infection, and does not require mycobacterial replication; moreover, it is characterized by a peculiar sequence of membrane modifications. In fact, membrane CD14 down-regulation, which normally mediates death signals in monocytes preceding the exposure of Annexin $V$ binding sites, ${ }^{4}$ is a later occurring membrane event in the course of MTB-induced apoptosis. ${ }^{3}$ The peculiarity of the apoptotic pathway followed by monocytes/macrophages after infection with high amounts of MTB could have important implications as only certain of these pathways have been associated with mycobacterial killing. ${ }^{5}$ In this context, monocyte/macrophage apoptosis has been reported to be associated with mycobacterial survival rather than to its killing. ${ }^{3}$

On these grounds, it would be of interest to identify the mycobacterial molecule/s delivering death signals in monocytes. This issue has previously been studied in other bacterial infections and some apoptogenic bacterial molecules have already been described. For example, Shigella flexnery, an enteric pathogen responsible for bacillary dysentery, induces both in vitro and in vivo macrophage apoptosis ${ }^{6,7}$ by directly activating caspase-1 through its binding with the invasion plasmid antigen (IPA) B. ${ }^{8}$ Similarly, Salmonella invasion protein B (SipB) has also been reported to mediate macrophage apoptosis $^{9}$ by binding to caspase-1. In these infections, caspase-1 mediated apoptosis allows efficient release of IL- $1 \beta$, which triggers acute inflammation. ${ }^{6-9}$ Other bacterial molecules, like cell wall associated mycobacterial lipoproteins, have more recently been shown to induce cell activation and apoptosis in THP-1 monocyte cell lines and this effect is mediated by Toll-like receptor $2 .{ }^{10}$ Members of these surface associated mycobacterial lipoproteins are the 19-kDa and $38-\mathrm{kDa}$ antigens, which are anchored in the cell wall by the lipid moiety and are expressed in the cell wall of MTB. While the biological function of the 19-kDa antigen is still unknown, the 38$\mathrm{kDa}$ antigen has been reported to work as a phosphate binding protein. ${ }^{11}$

In this study, we have investigated the role of cell wall associated $19-\mathrm{kDa}$ and $38-\mathrm{kDa}$ lipoproteins in apoptosis occurring in monocytes/macrophages at the early stages of infection with high amounts of MTB H37Rv, by using anti-mycobacterial $19-\mathrm{kDa}$ and anti-38-kDa monoclonal antibodies. Figure 1 shows increased levels of apoptosis in monocytes/macrophages, infected at a $\mathrm{MOI}$ of 20 with the virulent strain MTB $\mathrm{H} 37 \mathrm{Rv}$, already visible at $1 \mathrm{~h}$ after mycobacterial exposure in terms of both Annexin $\mathrm{V}$ (Figure 1A) and TUNEL (Figure 1B) positivity. In order to investigate the nature of the apoptogenic signal delivered by MTB H37Rv and the possible involvement of some mycobacterial wall lipoproteins in apoptosis induction, $40 \times 10^{6} \mathrm{CFU}$ of MTB H37Rv were preincubated for $15 \mathrm{~min}$ at $37^{\circ} \mathrm{C}$ and $5 \% \mathrm{CO}_{2}$ with $500 \mu \mathrm{g}$ of anti-19-kDa (clone HYT 6, mouse $\operatorname{lgG}_{1}$ ), or anti-38-kDa (clone HBT 12, mouse $\lg _{1}$ ) monoclonal antibodies suspended in $500 \mu \mathrm{l}$ of PBS. As control, bacilli were pre-incubated with the same amount of anti-HLA (Human Leukocyte Antigen)-DP (clone B7/21, mouse $\operatorname{IgG}_{1}$ ) monoclonal antibody. After incubation, bacterial suspensions were used to infect monocytes/macrophages as previously described. ${ }^{3}$ Flow-cytometry analysis was performed on monocytes/macrophages infected or not with 20 bacilli per cell of MTB H37Rv pre-incubated with HYT 6 , HBT 12 or B7/21 monoclonal antibodies. Figure 1C shows that the pre-opsonization of bacilli with anti$19 \mathrm{kDa}$ monoclonal antibody resulted in a strong inhibition of MTB-induced apoptosis (Figure 1C, filled histogram, $21 \%$ of Annexin $\mathrm{V}$ positive cells). A slightly lower percentage of Annexin $\mathrm{V}$ positive cells was observed in time-matched MTB 37Rv uninfected monocytes/macrophages (Figure 1C,D,E empty histograms, $17 \%$ of Annexin $V$ positive cells). The HYT 6-mediated effect resulted in an $80 \%$ inhibition when compared to the percentage of Annexin $\mathrm{V}$ positive monocytes/macrophages derived from MTB H37Rv infected and uninfected cultures (Figure 1A). Finally, the anti-19-kDa-mediated decrease of apoptosis appears to be specific. In fact, similar percentages of Annexin $\mathrm{V}$ positive cells were observed between monocytes/macrophages infected with untreated MTB H37Rv (Figure 1A) and monocytes/macrophages infected with MTB H37Rv either pre-opsonized with antimycobacterial $38 \mathrm{kDa}$ (Figure 1D, filled histogram, $36 \%$ of Annexin $V$ positive cells) or pre-incubated with anti-HLADP (Figure 1E, filled histogram, $34 \%$ of Annexin $V$ positive cells) monoclonal antibodies.

The present study identifies mycobacterial $19-\mathrm{kDa}$ lipoprotein as a key molecule mediating monocyte/ macrophage apoptosis induced by high amounts of MTB H37Rv at the early stages of infection. However, the fact that the anti-19-kDa mediated inhibition was not complete $(80 \%)$, suggests that other still unidentified mycobacterial molecules could be involved in this process. Our data are 

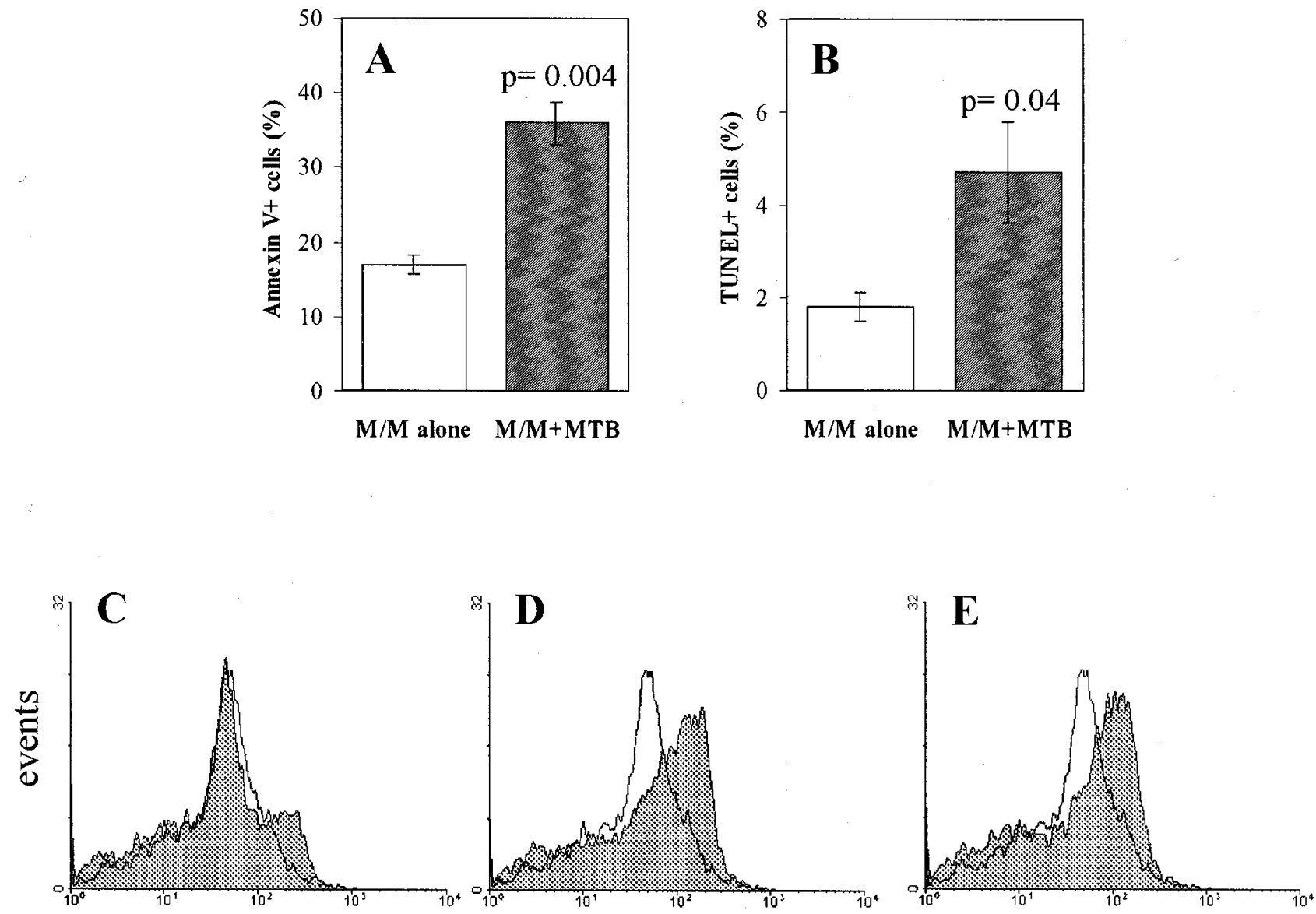

FITC-Annexin V

Figure 1 For all panels: peripheral blood mononuclear cells (PBMC) were isolated from human buffy coat blood preparations by centrifugation on ficoll-hypaque and suspended at $5 \times 10^{6} / \mathrm{ml}$ in complete medium [RPMI 1640 supplemented with $10 \%$ fetal bovine serum, $5 \mathrm{mM}$ L-glutamine and Gentramicine (5 $\left.\mu \mathrm{g} / \mathrm{ml}\right)$ ]. They were then incubated for $1 \mathrm{~h}$ in $5 \mathrm{ml}$ of complete medium at $37^{\circ} \mathrm{C}$ in polystyrene $T 25 \mathrm{~cm}^{2}$ tissue culture flasks (Corning, Cambridge, MA, USA). After incubation, nonadherent cells were removed by three washes with warm RPMI 1640. In each experiment, cells from a representative flask were analyzed in flow cytometry by CD14 staining and morphological parameters (forward scatter versus side scatter) to assess monocyte purity which was always more than $80 \%$. Adherencepurified monocytes/macrophages (M/M) in T25 $\mathrm{cm}^{2}$ tissue culture flasks were infected for $1 \mathrm{~h}$ with MTB H37Rv at a MOI of 20. Cells were collected, counted and monitored by flow-cytometry for apoptotic phenotype by Annexin V staining (A, C, D, E) and TUNEL (B), as previously described. ${ }^{3}$ Data from (A) and (B) are expressed as mean + S.E. derived from experiments performed on five different healthy donors and Student's $t$-test was used to compare means. (C) Monocytes/ macrophages were infected with MTB H37Rv pre-incubated for $15 \mathrm{~min}$ at $37^{\circ}$ with HYT 6 monoclonal antibody. (D) Monocytes/macrophages were infected with MTB H37Rv pre-incubated for $15 \mathrm{~min}$ at $37^{\circ} \mathrm{C}$ with HBT 12 monoclonal antibody. (E) Monocytes/macrophages were infected with MTB H37Rv pre-incubated for $15 \mathrm{~min}$ at $37^{\circ} \mathrm{C}$ with $\mathrm{B} 7 / 21$ monoclonal antibody. Panels $(\mathbf{C}-\mathbf{E})$ are representative of experiments performed on three different healthy donors. Monoclonal antibodies used in this study were kindly provided by Dr. M. Amicosante from the Laboratory of Clinical Pathology, IRCCS - L. Spallanzani Institute, Rome, Italy

in accordance with previously reported results showing that bacterial lipoproteins can mediate (i) cell activation and pro-inflammatory signals; ${ }^{12}$ and (ii) apoptosis by binding with Toll-like receptor $2 .^{10}$ In particular, IL-12 production, NF- $\kappa \mathrm{B}$ and iNOS activation in response to mycobacterial $19-\mathrm{kDa}$ has been reported in monocytes ${ }^{12}$ and a link between cell activation, as observed in terms of $\mathrm{NF}-\kappa \mathrm{B}$ activation, and apoptosis has been demonstrated following bacterial lipoprotein stimulation. ${ }^{10}$ In this context, cell activation and apoptosis can be induced by MTB infection depending upon the $\mathrm{MOI}$ used., ${ }^{3,13,14}$ In fact, previously reported results showed that low levels of $\mathrm{MTB}^{13}$ or $\mathrm{BCG}^{15}$ prevent monocyte apoptosis whereas high amounts of the virulent strain of MTB H37Rv can induce apoptosis. $^{2,3}$ It is plausible, therefore, to hypothesize that the presence of a threshold signal, represented by the amount of mycobacteria and possibly by the amount of cell wall associated 19-kDa lipoprotein, determines whether the cell will live or die.

The study was supported by MURST-CNR Biotechnology Program L. 95/95 and by II Research Program on AIDS (50B.10) of the ISS. 
A Ciaramella ${ }^{1}$, A Martino $^{1}$, Cicconi $^{1}$, V Colizzi $^{1,2}$ and M Fraziano*,1,2

${ }^{1}$ Department of Biology, University of Rome 'Tor Vergata', 00133 Rome, Italy ${ }^{2}$ International Centre for AIDS \& Emerging and Re-emerging Infections IRCCS

- L. Spallanzani Institute, Rome, Italy

${ }^{*}$ Corresponding author: Fax: 00-39-06-72594224;

E-mail: fraziano@bio.uniroma2.it

1. Weinrauch Y et al. (1999) Annu. Rev. Microbiol. 53: 155-187

2. Placido R et al. (1997) J. Pathol. 181: 31-38

3. Santucci M et al. (2000) J. Infect. Dis. 181: 1506-1509
4. Heidenreich S et al. (1997) J. Immunol. 159: 3178-3188

5. Kornfeld $\mathrm{H}$ et al. (1999) Cell Death Differ. 6: 71-78

6. Zychlinsky A et al. (1992) Nature 358: 167-169

7. Zychlinsky A et al. (1996) Infect. Immun. 64: 5357-5365

8. Hilbi H et al. (1998) J. Biol. Chem. 273: 32895-32900

9. Hersh D et al. (1999) Proc. Natl. Acad. Sci. USA 96: 2396-2401

10. Aliprantis AO et al. (1999) Science 285: 736-739

11. Espitia $C$ et al. (1992) Infect. Immun. 60: 2998-3001

12. Brightbill HD et al. (1999) Science 285: $732-736$

13. Durrbaum-Landmann I et al. (1996) Infect. Immun. 64: 5384-5389

14. Fraziano M et al. (1999) AIDS Res. Human Retrovir. 15: 869-874

15. Kremer L et al. (1997) Eur. J. Immunol. 27: 2450-2456 